\title{
Mood Moderates the Effect of Aesthetic Appeal on Performance.
}

\author{
Irene Reppa \\ Swansea University, United Kingdom \\ Siné McDougall \\ Bournemouth University, United Kingdom \\ Andreas Sonderegger \\ University of Fribourg, Switzerland \\ William C. Schmidt \\ SR Research, Canada
}

Running head: Mood and Aesthetic Appeal

Correspondence: For all correspondence regarding this manuscript please contact Irene Reppa, Department of Psychology, Swansea University, SA2 8PP, UK. Email:

i.reppa@swansea.ac.uk. 


\begin{abstract}
Aesthetically appealing stimuli can improve performance in demanding target localisation tasks compared to unappealing stimuli. To examine the possible underlying mechanism mediating the effects of appeal on performance, participants were put in a positive or negative mood prior to carrying out a visual target localisation task with appealing and unappealing targets. Positive mood initially led to faster localisation of appealing compared to unappealing stimuli, while an advantage for appealing over unappealing stimuli emerged over time in negative mood participants. The findings are compatible with the idea that appealing stimuli may be inherently rewarding, with mood temporarily gating any rewarding effects that aesthetic appeal might have on performance. However, this gating by mood is temporary and aesthetic appeal help counteract the adverse effect of negative mood on performance.
\end{abstract}

Keywords: aesthetic appeal, mood, reward, performance, icons 
Aesthetic appreciation is a fundamental emotion that influences our daily behaviour (e.g., Schindler, Hosoya, Menninghaus, et al., 2017). We actively seek to surround ourselves with objects that we find pleasing to our senses. We go to art galleries for new aesthetic experiences and bring beautiful things into our homes. Interestingly, when we look at something aesthetically pleasing, it can compel us to interact with it, and make us want to spend time with it.

Aesthetic appeal is used to refer to mild aesthetic experiences and is revealed by simple rating judgments made on the basis of liking (see Reber, Schwarz \& Winkielman, 2004 for review). Far from being all window-dressing, aesthetic appeal influences not only our behaviour but also our performance with the objects around us. Dealing with appealing stimuli can increase performance efficiency relative to unappealing stimuli in tasks requiring fast searches (e.g., Moshagen, Musch, \& Göritz, 2009; Reppa \& McDougall, 2015; Sonderegger \& Sauer, 2010). This effect is particularly apparent when dealing with hard to find stimuli (e.g., Moshagen et al., 2009; Reppa \& McDougall, 2015).

What might account for improved performance with appealing stimuli? One possibility is that appealing stimuli might be affectively positive stimuli. Different lines of evidence suggest this as a reasonable suggestion. Aesthetic appeal as a visual attribute of the world around us is perceived extremely quickly - within 50 milliseconds (e.g., Lindgaard, Fernandes, Dudek, \& Brown, 2006) - rendering it a good candidate to influence time-critical performance. Evidence from human-computer interaction and consumer research has shown that appealing stimuli are seen as more positive, by 
virtue of their ability to elicit a positive attitude in the observer (e.g., Sonderegger, Zbinden, Uebelbacher, \& Sauer, 2012; Porat \& Tractinsky, 2012; Thuring \& Mahlke, 2007). In the field of marketing, attempts to influence consumers' affect through appealing design are common (e.g., Bloch, 1995; Kotler \& Rath, 1984; Whitney, 1988).

Another possible mechanism through which appeal might exert its influence on performance is reward. Evidence from neuroimaging studies shows that aesthetic judgment is correlated with activity in neural systems underlying reward, both for auditory (e.g., Blood \& Zatorre, 2001) and visual stimuli (e.g., Aharon, Etcoff, Ariely, Chabris, O'Connor, \& Breiter, 2001; Kawabata \& Zeki, 2004; Kirk, Skov, Hulme, Christensen, \& Zeki, 2009; Leder, Belke, Oeberst, \& Augustin 2004; Vartanian \& Goel, 2004). Therefore, the potentially rewarding value of aesthetically pleasing stimuli may be implicated in the beneficial effect of appeal on performance.

The manipulation of mood is well-suited to provide specific insight into the way appealing stimuli might influence performance. A survey of experimental work on mood and performance reveals that mood can greatly influence performance efficiency in a variety of tasks by altering the way people attend to their environments (e.g., Raila, Scholl \& Gruber, 2015; Rowe, Hirsh, \& Anderson, 2007). Compared to individuals in negative moods, those in positive moods take less time to complete certain tasks (e.g., Mayer \& Bremer, 1985; Schmitz, De Rosa, \& Anderson, 2009), focus on global as opposed to local details of an object or scene (e.g., Gasper \& Clore, 2002), and exhibit a wider attentional focus, as shown using methods from psychophysics (e.g., Rowe et al., 2007; but see Bruyneel, van Steenbergen, Hommel, Band, De Raedt, \& Koster, 2013 for 
mixed findings), eye-tracking (e.g., Wadlinger \& Isaacowitz, 2006) and neuro-imaging (e.g., Schmitz et al., 2009).

If appealing and unappealing stimuli are affective stimuli, then they might be expected to act like other affective stimuli in studies manipulating mood. Evidence from work on how mood might influence attentional processing, has shown that the current mood of the observer interacts with affectively valenced cues to bias attention towards congruent environmental stimuli to the detriment of other stimuli. Some studies find that participants' attention can be biased towards stimuli that are more congruent with their mood (e.g., Becker \& Leinenger, 2011; Wadlinger \& Isaacowitz, 2006).

Alternatively, there is evidence showing a mood-incongruent attentional bias that is hypothesised to maintain homeostasis (e.g., Derryberry \& Tucker, 1994; Gawronski, Deutsch, \& Strack, 2005; Rothermund, Wentura,\& Bak, 2001; Rothermund, Voss, \& Wentura, 2008). According to the counter-regulation principle of affective processing, attention is allocated to information that is incongruent to the current affective and motivational state (e.g., Rothermund, et al., 2008). Critically, counter-regulatory processes are triggered if the affective state is strong enough, but in weak affective states mood congruency effects are more likely to be observed, which are thought to be related to semantic priming by congruent affective information (e.g., Schwager \& Rothermund, 2014).

Therefore, if appealing and unappealing stimuli are affective stimuli, we might expect that participants might attend preferentially to stimuli that are of the opposite valence to the induced mood state (assuming that the induced state is sufficiently 
strong). For instance, in an induced negative mood state, appealing stimuli may yield a localisation advantage over unappealing stimuli, and vice versa for an induced positive mood state.

Mood is known to moderate the effects of rewarding stimuli on performance,. For instance, for participants in a positive mood, rewarding words (e.g., Tamir \& Robinson, 2007), or wins in a simple gambling game (e.g., Foti \& Hajcak, 2010) are more likely to be preferentially processed than their non-rewarding counterparts. However, under negative mood states, attention is biased more towards mood-incongruent stimuli, presumably as part of a regulatory mechanism to repair the elicited negative mood (e.g., Isaacowitz, Toner, \& Neupert, 2009; Sanchez, Vazquez, Gomez, \& Joormann, 2014). It has been proposed that positive mood states may facilitate a selective focus on rewarding or desirable features of the environment (i.e., appealing icons in our study), which may facilitate the attainment of positive outcomes (e.g., Tamir \& Robinson, 2007). In contrast, under negative mood states a focus on rewarding information may serve to repair negative mood (e.g., Sanchez et al., 2014). If reward is implicated in performance with aesthetic stimuli, then aesthetically pleasing icons would be preferentially attended to and localized faster compared to unappealing icons, for both mood groups (see Table 1 for predictions).

In two experiments participants were induced into either a positive or negative mood state, following which they completed a target localisation task using a wellcontrolled and previously used icon set (e.g., McDougall, Curry, \& de Bruijn, 1999). As the effects of mood induction have been shown to wane during the course of the 
experiment, (e.g., Brandenburg \& Backhaus, 2016; Sauer \& Sonderegger, 2009), performance was examined over time. The different predicted outcomes are outlined in Table 1.

TABLE 1 ABOUT HERE

\section{Experiment 1 Method}

\section{Participants}

Forty Swansea University undergraduates took part in the experiment in exchange for course credit, with 20 participants per group. Twenty students (10 females) aged between 18 and $20(M=19.21, S D=0.71)$ participated in the negative mood condition, and twenty (18 females) aged between 19 and $24(M=21.05, S D=1.54)$ were assigned randomly to the positive mood condition.

\section{Apparatus \& Materials}

The experiment was run using PsyScope (Cohen, MacWhinney, Flatt, \& Provost, 1993) on a Mac mini computer. The music for the mood induction phase was delivered via headphones connected to the computer and all visual stimuli were presented on the 19" screen monitor.

The mood induction procedure used was created and validated by Robinson, Grillon, and Sahakian (2012) and lasted 15 minutes. The negative mood induction 
consisted of reading 60 sentences, each presented for 12 seconds in white ink appearing on a blue background accompanied by the Adagio for strings, Op. 11 by Samuel Barber. The sentences had progressively negative meaning starting with sentences such as "I am feeling a little down today" and progressing to more emotionally charged sentences including, "I just don't care about anything. Life just isn't any fun”.

For the positive mood induction, each sentence appeared for 12 seconds in pale red writing on a yellow background and was accompanied by the Brandenburg Concerto No 3 by Hubert Laws. They were progressively positive in content, starting with "I feel light-hearted" and moved onto "This is just one of those days when I'm ready to go!".

A 9-point mood rating scale was presented on paper for participants to complete (Bradley \& Lang, 1994). The entire mood-rating instrument measures three dimensions of affect, i.e., valence, arousal, and dominance. The current study used the dimension of valence only.

Stimuli for the target localisation task were the same black-and-white icons used in Reppa and McDougall (2015) and were carefully controlled using ratings from an icon corpus (McDougall \& Reppa, 2008; McDougall, Curry \& de Bruijn, 1999). Appeal ratings from the corpus differed significantly for the appealing and unappealing icons (Figure 1A). Importantly, the visual complexity, concreteness and familiarity of appealing and unappealing icons were carefully matched because these icon characteristics are known to affect icon search performance (see Isherwood \& McDougall, 2009). Symmetry was also controlled with about half of appealing and half of the unappealing stimuli being mirror-symmetrical. Thus, any effects observed would be the result of either differences 
in icon appeal or the mood manipulation. Full details for the icons used here are reported in our earlier work (see Reppa \& McDougall, 2015, Exp.1).

\section{FIGURE 1 ABOUT HERE}

\section{Design and Procedure}

The experiment was based on a 2 Mood (positive vs. negative) X 2 (Appeal: appealing vs. unappealing) X 2 (Complexity: complex vs. simple) X 3 (Block: 1, 2, \& 3) mixed design, with Mood manipulated between-participants. There were 360 trials per participant, presented in 3 blocks with breaks in-between. There were 120 trials in each block, where each of the two icon types appeared in random order and randomly within all of the 9 possible array locations. Across the 360 trials, each icon was presented nine times as a target and 72 times as a distractor. The dependent measure was response time (RT).

The mood valence rating scale was used to rate mood state at the start of the study. Mood ratings could vary from $1=$ very negative feelings, to $9=$ very positive feelings, which participants marked accordingly with a pen. Following this initial recording of mood state, the mood induction procedure lasted for 15 minutes, following which participants were administered a second mood rating scale to allow us to determine if a mood alternation had been accomplished. 
The target localisation task was identical to Reppa and McDougall (Exp.1; 2015). To start each trial, participants used the mouse to click an "OK" button on the bottom left corner of the computer screen (Figure 1B). The target icon was then presented alone for 2 seconds at the top left corner of the screen. Following target offset participants clicked once again on the "OK" button to trigger the presentation of the 9-icon array. This ensured that participants started each trial with the mouse pointer at the same point on the display. Participants had to click on the target icon as quickly as possible. The same process was repeated for 360 trials, with each icon shown 9 nine times, once in each position in the array. The remaining of the array consisted of 2 complex appealing, 2 complex unappealing, 2 simple appealing and 2 simple unappealing icons, all drawn from the same 40 icons, with the constraint that the target did not appear also as a distractor in the same trial. Incorrect responses received a 500-ms beep sound. Finally, a third and final mood-rating scale was administered to obtain a final mood rating at the end target localisation task.

\section{Experiment 1 Results}

\section{Data processing}

One participant's data was removed from the negative mood group due to software malfunction, which was realised after the data was processed, and one participant's data was removed from the positive mood group analysis because their response times exceeded 3 standard deviations from the group's mean response times. Including this participant's data did not change the pattern or the significance of results reported below. 
In the negative mood group, 3.5\% of trials were errors, which were removed from the analysis, and not analysed further. Furthermore, $0.8 \%$ of the correct trials were longer than 3 seconds and were removed from the data and not analysed further. In the positive mood group, $3.6 \%$ of the total number trials were errors and were removed from the data and not analysed further. In $0.9 \%$ of the correct trials the response time was longer than 3 seconds, and those trials were removed from the data.

\section{Mood Induction Manipulation Check}

Mean mood ratings before the mood induction, immediately after the mood induction, and at the end of the localisation task appear in Figure 2 separately for the two mood groups. A 2 (Group: positive vs. negative mood) X 3 (Time: before, after, \& final) mixed ANOVA with repeated measures on the Time variable, revealed a significant main effect of Group, $F(1,36)=18.84, p<.001$, partial $\eta^{2}=.14$, and a significant main effect of Time, $F(2,72)=26.21, p<.001$, as well as a significant interaction, $F(2,72)=26.69, p<.001$. As expected, there was no difference in mood ratings between the positive and negative groups before the mood induction, $t(36)=1.03, p>.05$, but the positive group gave higher mood ratings than the negative group both immediately after the mood induction and at the end of the task $[t(36)=6.31, p<.0001$, and $t(36)=3.45, p<.001$, respectively]. 
To ensure that the mood manipulation worked for both groups two separate pairwise comparisons were carried out between mood ratings before the mood induction and immediately after the mood induction. For the positive mood group, there was a significant increase in positive mood from before $(M=6.10, S D=1.20)$ to after the mood induction $(M=7.42, S D=1.12), t(18)=8.54, p<.0001$, confirming that the positive mood manipulation worked. Similarly, for the negative mood group, there was a significant decrease in mood from before $(M=5.73, S D=.99)$ to after the mood induction $(M=4.57, S D=1.60), t(18)=3.54, p=.002$, confirming that the negative mood manipulation worked as expected.

Next, we examined how mood ratings changed from immediately after the mood induction to mood ratings at the end of the localisation task. For the positive mood group, mood ratings significantly decreased from being very high right after the mood induction $(M=7.42, S D=1.12)$ to a more neutral point on the scale at the end of the task $(M=5.58, S D=1.26), t(18)=11.67, p<.001$. For the negative mood group there was no difference in mood ratings between right after the mood induction $(M=4.57, S D=1.61)$ and those taken at the end of the task $(M=4.21, S D=1.18), t(18)=1.38, p>.05$, suggesting that negative mood was maintained throughout the task. So, overall, participants in the positive group reported a more positive mood after the mood induction, which was reduced back to baseline by the end of the experiment, while participants in the negative mood group reported a negative mood at both time points after the mood induction. 
Response Time (RT) Analysis

Cell means appear in Figure 3. A 2 Mood (positive vs. negative mood) X 2 (Complexity: simple vs. complex) X 2 (Appeal: appealing vs. unappealing) $\times 3$ (Block: 1, 2, 3) mixed ANOVA was carried out on correct response times with repeated measures on Complexity, Appeal, and Block. The main effect of Mood was significant, $F(1,36)=6.42$, $p=.02$, partial $\eta^{2}=.151$, with slower RT in the negative mood group. There was a significant main effect of Complexity $F(1,36)=149.21, p<.0001$, partial $\eta^{2}=.806$ with simple icons found faster than complex icons. The main effect of Appeal was significant $F(1,36)=9.74, p=.004$, partial $\eta^{2}=.130$, with appealing icons found faster than unappealing icons. There was a significant main effect of Block, $F(2,72)=6.33, p=.003$, partial $\eta^{2}=.186$, with RT decreasing across the 3 blocks of trials. There was a significant Block X Appeal X Mood interaction $F(2,72)=3.19, p=.047$, partial $\eta^{2}=.08$, and a significant Appeal X Complexity interaction $F(1,36)=14.48, p<.001$, partial $\eta^{2}=.09$. The 4-way interaction was also significant, $F(2,72)=3.76, p=.028$, partial $\eta^{2}=.08$.

Two separate ANOVAs were carried out to examine the 4-way interaction. Given the significant Appeal X Complexity interaction observed here and in our previous work (e.g., Reppa \& McDougall, 2015), we examined simple and complex icons separately. A mixed 2 (Mood: positive vs. negative) X 2 (Appeal: appealing vs. unappealing) X 3 (Block: $1,2, \& 3)$ mixed ANOVA on simple icons RT, revealed only a significant main effect of Mood, $F(1,36)=6.66, p=.01$, partial $\eta^{2}=.156$, with faster $\mathrm{RT}$ in the positive compared to the negative mood group. There were no other significant main effects or interactions. 
A second repeated-measures ANOVA examined the effect of Appeal, Block and Mood Condition for complex icons RT, showed a significant main effect of Mood, $F(1$, $36)=5.46, p=.02$, partial $\eta^{2}=.132$, a significant main effect of Appeal $F(1,36)=19.44, p<$ .001 partial $\eta^{2}=.286$, and a significant main effect of Block $F(2,72)=7.65, p<.001$, partial $\eta^{2}=.207$. The three-way interaction was also significant, $F(2,72)=5.67, p<.001$ partial $\eta^{2}=.277$. For participants in the positive mood condition, appealing complex icons were localised faster than unappealing complex icons in Block $1, t(18)=4.209, p<.001$, and in Block 2, $t(18)=2.35, p=.03$, but not in Block 3, $t(18)=1.08, p=.40$. In stark contrast, for participants in the negative mood condition, there was no difference in response times between appealing and unappealing complex icons in Block 1, $t(18)=.47, p=.65$, or in Block 2, $t(18)=1.96, p=.12$, but appealing complex icons were found significantly faster than unappealing complex icons in Block $3, t(18)=2.791, p=.01$. No other interactions reached significance.

FIGURE 3 ABOUT HERE

\section{Experiment 1 Discussion}

Experiment 1 examined how appeal might exert its influence on performance by manipulating mood. Mood clearly moderated the effect of appeal on performance. For participants in a positive induced mood processing of aesthetically pleasing icons was immediately enhanced, but this advantage disappeared by the end of the session as 
response latencies for complex unappealing stimuli improved with exposure. In contrast, participants in a negative mood only showed enhanced processing of aesthetically appealing stimuli over time, while response times to unappealing stimuli remained constant through the session. The findings are discussed in detail in the General Discussion,

\section{Experiment 2}

Experiment 2 was designed to conceptually replicate Experiment 1 and use a different mood manipulation to examine the generality of findings in Experiment 1 . The hypotheses and predictions were the same as those of Experiment 1.

\section{Experiment 2 Method}

\section{Participants}

Forty-five participants took part in Experiment 2 in exchange of participant pool credits. In the negative mood group there were 23 participants between the ages 18 and 24 $(M=20.00, S D=1.89), 16$ females and 7 males. In the positive mood group 22 participants took part, 19 females and 3 males, aged between 19 and $22(M=20.56, S D=.96)$.

\section{Apparatus \& Materials}

For the mood induction a mixed procedure was used. The music mood induction procedure (MIP) and the imagination MIP (e.g., Schwarz \& Clore, 1983) with the added instruction of writing down a story for strengthening the induction. Negative mood was induced by asking participants to write a sad imaginary or real story while listing to 
music in a minor key, Adagio for strings, Op. 11 by Samuel Barber, for 6 minutes. For the positive mood induction, a happy imaginary or real story writing task was used accompanied with music in a major key, Piano Concerto No. 4, Op. 58 in G Major: III. Rondo: Vivace by Ludwig van Beethoven for 6 minutes. In both conditions the writing task took place for as long as the music played. The story was written on paper with a black or blue pen.

The Self-Assessed-Manikin (SAM) scale measured arousal and valance. For the arousal scale, 1 means very calm and 9 very anxious. In the valance scale, 1 means very unpleasant and 9 very pleasant.

For the target localisation task, the same apparatus and icon stimuli were used as in Experiment 1.

Design

The experiment was based on a 2 (Mood: positive vs. negative) X 2 (Appeal: appealing vs. unappealing) $\times 2$ (Complexity: complex vs. simple) mixed design with mood manipulated between participants. There were 360 trials per participant, presented in 3 blocks with break-ins between. There were 120 trials in each block where each of two icon types (complex appealing and unappealing icons) appeared in random order and randomly within all of the nine possible array locations. Across the 360 trials, each icon (both experimental and filler) was presented 9 times as a target and 72 as a distractor. The dependant measure was response time (RT). 


\section{Procedure}

At the start of the experiment, participants were asked to complete the valence and arousal SAM scales. After that a music piece (sad or happy, depending on group allocation) started playing while they wrote a real or imaginary story (sad or happy, depending on group allocation) for 6 minutes. The mood measurements as above followed immediately after the mood induction. The target localisation task followed, which was identical to that in Experiment 1. At the end of the localisation task, participants completed the two mood measures described above for one last time.

\section{Experiment 2 Results}

\section{Mood Induction Manipulation Task}

Mean valance and arousal ratings before the mood induction, immediately after the mood induction, and at the end of the localisation task appear in Figure 4 separately for the two mood groups.

\section{FIGURE 4 ABOUT HERE}

For arousal there was a significant main effect of Time, $F(2,86)=5.72, p=.005$, $\eta_{\text {partial }}{ }^{2}=.12$, and a significant interaction, $F(2,86)=10.27, p<.001$, partial $\eta^{2}=19$. Pairwise were carried out to examine the interaction. In the positive mood group, arousal significantly increased after the mood manipulation, [time 1 vs. time $2, t(21)=4.96$, $p<.001$ ], while significantly decreasing again to pre-induction levels at the last 
measurement, [time 2 vs. time $3, t(21)=4.54, p<.0001$ ]. In contrast, in the negative mood group, arousal significantly decreased following the mood manipulation [time 1 vs. time $2, t(22)=2.76, p=.01$ ], and stayed low at time 3, [time 2 vs. time $3, t(22)=.69, p=.49$ ].

For valance ratings, there was a significant main effect of Time $F(2,86)=12.95$, $p<.0001$, partial $\eta^{2}=.23$, and a significant interaction, $F(2,86)=7.54, p<.001, \eta_{\text {partial }}{ }^{2}=.15$. In the positive mood group, mood marginally increased after the mood manipulation, [time 1 vs. time $2, t(21)=1.89, p=.07]$, while significantly decreasing again to preinduction levels, [time 2 vs. time $3, t(21)=4.17, p<.0001$ ]. In contrast, in the negative mood group, valence significantly decreased following the mood manipulation [time 1 vs. time $2, t(22)=3.71, p<.001$ ], and stayed low at time 3 , [time 2 vs. time $3, t(22)=.72$ $p=.48]$. Thus, overall, the combined mood induction used in Experiment 2 influenced ratings of arousal for both positive and negative mood states, and ratings of valence, especially for the negative mood state.

\section{Response Time (RT) Analysis}

Outlier RTs that were less that 150 milliseconds or greater than 3 seconds accounted for $1.28 \%$ in the positive and $1.68 \%$ in the negative mood groups. Those were removed from the correct RT analysis. Errors accounted for $1.34 \%$ of all trials in the positive mood group and $1.63 \%$ of all trials in the negative mood group. Those were removed and not analysed further.

FIGURE 5 ABOUT HERE 
Cell means appear in Figure 5 for positive and negative group respectively. A 2 (Mood: positive vs Negative) X 3 (Block 1, 2, \& 3) X 2 (Appeal: appealing vs unappealing) X 2 (Complexity: simple vs complex) mixed ANOVA on correct RT, with repeated measures on Block, Appeal, and Complexity was carried out. There was a significant effect of Block, $F(2,86)=32.77, p<.0001, \eta_{\text {partial }}{ }^{2}=.43$, with RT decreasing significantly from Block 1 to Block $2 t(44)=4.39, p<.001$, and from Block 2 to Block $3 t(44)=4.68$, $p<.001$. There was also a significant main effect of Complexity $F(1,43)=272.51, p<.0001$, partial $\eta^{2}=.86$, with faster RT for simple compared to complex icons. Icon Appeal did not have a significant main effect on $\mathrm{RT}, F(1,43)=12.36, p=.13$, but it was involved in a significant Appeal X Complexity interaction, $F(1,43)=16.79, p<.0001, \eta_{\text {partial }}{ }^{2}=.28$. Pairwise tests showed no difference between simple appealing and unappealing icons, $t(44)=1.87, p=.07$, while complex appealing icons were localised faster than complex unappealing icons, $t(44)=2.27, p=.03$.

Finally, there was significant 3-way interaction between Mood, Block, and icon Appeal, $F(2,86)=6.91, p=.002, \eta_{\text {partial }}{ }^{2}=.14$. For participants in the positive mood condition, appealing icons were localised faster than unappealing icons in Block 1, $t(21)=3.12, p=.005$, but there was no difference in Block $2, t(21) .626, p=.55$, or in Block $3, t(21)=1.35, p=.19$. In contrast, for participants in the negative mood condition, there was no difference in response times between appealing and unappealing icons in Block $1, t(22)=.50, p=.62$, or in Block 2, $t(22)=1.13, p=.27$, but appealing icons were localised faster than their unappealing counterparts in Block $3, t(22)=3.18, p=.004$. 
There were no other significant interactions.

FIGURE 5 ABOUT HERE

\section{General Discussion}

The aim of the current study was to examine whether appealing stimuli can act like affective stimuli. Some evidence for this notion comes from studies in humancomputer interaction (e.g., Sonderegger et al., 2012; Porat \& Tractinsky, 2012; Thuring \& Mahlke, 2007) showing... Two potential outcomes were expected if appealing stimuli act like affective stimuli. Appealing and unappealing stimuli may have elicited mood congruent effects in performance whereby participants in a positive induced mood state would preferentially attend to appealing over unappealing stimuli, and vice versa for those in an induced negative mood state. Mood congruency effects have been observed elsewhere with emotional stimuli, such as happy or sad faces, following mood manipulations (e.g., Becker \& Leinenger, 2011; Wadlinger \& Isaacowitz, 2006). Alternatively, appealing and unappealing stimuli may have elicited mood-incongruent effects, whereby participants in a positive mood would preferentially attend to unappealing more so than appealing stimuli, while those in an induced negative mood would be biased more towards appealing stimuli.

Appeal interacted with participant mood to influence the pattern of results. Participants in a positive mood showed initially an advantage for appealing stimuli, 
which dissipated over time, likely as a result of dissipating mood. However, the lowered mood at the last block of trials did not lead to an advantage for unappealing stimuli as would be predicted if appealing stimuli acted as affective stimuli. Meanwhile, participants in a negative mood showed no initial advantage for unappealing stimuli, but instead an advantage for appealing stimuli eventually emerged.

The pattern of results could suggest that appealing stimuli act as affective stimuli. The advantage of appealing stimuli in the negative mood group is consistent with the counter-regulation principle - participants (eventually) show preferential processing for appealing over unappealing stimuli. Meanwhile, the absence of this incongruency effect in the positive mood group could be attributed to less potent affect in this group. Indeed, in the positive mood condition the induced affective state dissipated towards the end of the experiment and was less intense than the negative affective state which lasted longer in both experiments. There is evidence that counter-regulatory incongruency effects are only observed if the affective state is strong enough (e.g., Schwager \& Rothermund, 2014) ${ }^{1}$.

Generally, mood manipulations are not always successfully at triggering counterregulatory processes, as they tend to induce a mood, which is a diffuse, background affective state and not directly linked to a specific emotion (see Schwager \& Rothermund, 2013, for discussion). Furthermore, as we did not manipulate mood state strength, what evidence do we have that the positive mood was weak and the negative

\footnotetext{
${ }^{1}$ We thank a reviewer for this suggestion.
} 
one strong? Both mood induction procedures employed in the current study (i.e., Exp. 1 Velten sentences along with music; Exp.2 imagination and music) are thought to be more effective at inducing negative as opposed to positive mood states (e.g., Westermann, Spies, Stahl, \& Hesse, 1996). In Experiment 1, while both mood inductions were effective, the negative mood induction lasted for longer (until the last mood measurement right after the experiment). Similarly, in Experiment 2, the negative mood induction effectively induced negative mood and low arousal, both of which lasted until the end of the study, while the positive mood induction increased arousal but only marginally improved mood. Thus, overall, the effects of the negative mood induction were stronger and lasted for longer than those of the positive mood induction. The counter-regulation principle can largely account for the current pattern of results: when the mood was not strong enough, mood congruency effects were observed, whole under negative mood, which was stronger, mood-incongruent effects were observed.

Although our findings suggest that appealing stimuli can be affectively positive (yielding mood-congruent effects in the weaker positive mood state, and moodincongruent effects in the stronger negative mood state), we did not find any evidence that unappealing icons might be affectively negative - that is, no evidence for mood incongruency effects in the positive mood group, whereby participants would preferentially attend to unappealing stimuli. This could be due to the fact that such counter-regulatory effects are triggered by strong emotional states rather than mood states. As the positive mood state was relatively weaker than the negative one, it would be unlikely to trigger mood-incongruency effects. Future work should systematically 
examine the counter-regulatory effects of appealing stimuli after manipulating affectivemotivational states, as opposed to mood states.

Another likely mechanism by which appeal might influence performance is reward (e.g., Kirk et al., 2009). That is, appealing stimuli may act as rewarding stimuli, while unappealing stimuli may be reward-neutral. Previous evidence has shown that rewarding stimuli are preferentially attended to both under positive (e.g., happy) moods biasing attention towards rewarding stimuli (e.g., Tamir \& Robinson, 2007), and under negative (e.g., sad) moods (e.g., Lerner et al. 2004; Raghunathan \& Pham 1999). The current findings suggest that appealing stimuli may have a reward value associated with them: in both experiments appealing stimuli were processed faster than unappealing ones. At the start of the experimental trials, participants in a positive mood were biased toward detecting aesthetically pleasing images, while participants in a negative mood were indifferent. However, with exposure, the indifference of participants in negative mood towards appealing stimuli turned into a performance benefit. This benefit for appealing icons was not simply an effect of experience with the task, as unappealing icons remained slow to be detected and performance did not benefit from task experience as much as with appealing icons. The benefit for appealing over unappealing icons was likely related to two factors. One was the persistent low mood of participants - mood remained low throughout the experiment for negative mood participants. The strong negative mood manipulation made it more likely that attention would be directed to mood-incongruent stimuli, that are more rewarding. Second, the emerging benefit for appealing icons in negative mood participants was likely to be a result of the 
reinforcing power of aesthetically pleasing stimuli, which builds over time (e.g., Mackintosh, 1975). Future studies should directly test the hypothesis that appealing stimuli are indeed rewarding in a similar way that money might be (e.g., Anderson, Laurent, \& Yantis, 2011).

In conclusion, the current study has shown that aesthetic appeal dynamically influences performance even when irrelevant to the task and it interacted with mood to determine performance efficiency - when in a positive mood appeal led to the best performance, while it helped beat the detrimental effects of negative mood on performance - eventually.

The current findings have practical implications in the field of marketing and retail. We have often walked in stores where music, objects, and scents have been specially selected to influence our buying behaviour. Here we show why and how this practice works: being in a positive mood makes us more likely to engage with things we like. But even in a negative mood state, appealing items are likely to capture attention and consequently influence behaviour - as long as we remain exposed to aesthetically appealing stimuli long enough. 
Mood and Aesthetic Appeal

Acknowledgments: We are grateful to Ben Yaxley, Menai Richards and Maria Papastavraki for help with data collection. 


\section{References}

Aharon, I., Etcoff, N., Ariely, D., Chabris, C. F., O'Connor, E., \& Breiter, H. C. (2001). Beautiful faces have variable reward value: $\mathrm{fMRI}$ and behavioral evidence. Neuron, 32(3), 537-551.

Anderson, B. A., Laurent, P. A., \& Yantis, S. (2011). Value-driven attentional capture. Proceedings of the National Academy of Sciences, 108(25), 10367-10371.

Becker, M. W., \& Leinenger, M. (2011). Attentional selection is biased toward moodcongruent stimuli. Emotion, 11(5), 1248-1254.

Blood, A. J., \& Zatorre, R. J. (2001). Intensely pleasurable responses to music correlate with activity in brain regions implicated in reward and emotion. Proceedings of the National Academy of Sciences, 98(20), 11818-11823.

Bradley, M. M., \& Lang, P. J. (1994). Measuring emotion: the self-assessment manikin and the semantic differential. Journal of Behavior Therapy \& Experimental Psychiatry, 25(1), 49-59.

Brandenburg, S., \& Backhaus, N. (2016). The dynamics of film-induced affect and its effect on the interaction with tablet PCs. Behaviour \& Information Technology, 35(5), 410-421.

Bruyneel, L., van Steenbergen, H., Hommel, B., Band, G. P., De Raedt, R., \& Koster, E. H. (2013). Happy but still focused: failures to find evidence for a mood-induced widening of visual attention. Psychological Research, 77(3), 320-332. 
Cohen, J., MacWhinney, B., Flatt, M., \& Provost, J. (1993). PsyScope: An interactive graphic system for designing and controlling experiments in the psychology laboratory using Macintosh computers. Behavior Research Methods, Instruments, \& Computers, 25(2), 257-271.

Fredrickson, B. L., Mancuso, R. A., Branigan, C., \& Tugade, M. M. (2000). The undoing effect of positive emotions. Motivation \& Emotion, 24, 237-258.

Foti, D., \& Hajcak, G. (2010). State sadness reduces neural sensitivity to nonrewards versus rewards. Neuroreport, 21(2), 143-147.

Gasper, K., \& Clore, G. (2002). Attending to the Big Picture: Mood and Global Versus Local Processing of Visual Information. Psychological Science, 13(1), 34-40.

Gawronski, B., Deutsch, R., \& Strack, F. (2005). Approach/avoidance-related motor actions and the processing of affective stimuli: Incongruency effects in automatic attention allocation. Social Cognition, 23(2), 182-203.

Isaacowitz, D. M., Toner, K., \& Neupert, S. D. (2009). Use of gaze for real-time mood regulation: Effects of age and attentional functioning. Psychology \& Aging, 24(4), 989. 
Kawabata, H., \& Zeki, S. (2004). Neural correlates of beauty. Journal of Neurophysiology, 91(4), 1699-1705.

Kirk, U., Skov, M., Hulme, O., Christensen, M. S., \& Zeki, S. (2009). Modulation of aesthetic value by semantic context: An fMRI study. Neuroimage, 44(3), 1125-1132.

Leder, H., Belke, B., Oeberst, A., \& Augustin, D. (2004). A model of aesthetic appreciation and aesthetic judgments. British Journal of Psychology, 95(4), 489-508.

Lindgaard, G., Fernandes, G., Dudek, C., \& Brown, J. (2006). Attention web designers: You have 50 milliseconds to make a good first impression! Behaviour \& information technology, 25(2), 115-126.

Mackintosh, N. J. (1975). A theory of attention: variations in the associability of stimuli with reinforcement. Psychological Review, 82(4), 276.

McDougall, S., \& Reppa, I. (2008). Why do I like It? The Relationships between Icon Characteristics, user Performance and Aesthetic Appeal. Proceedings of the Human Factors \& Ergonomics Society Annual Meeting, 52(18), 1257-1261.

McDougall, S., Curry, M., \& de Bruijn, O. (1999). Measuring symbol and icon characteristics: Norms for concreteness, complexity, meaningfulness, familiarity, and semantic distance for 239 symbols. Behavior Research Methods, Instruments, \& Computers, 31(3), 487-519. 
Moshagen, M., Musch, J., \& Göritz, A. (2009). A blessing, not a curse: Experimental evidence for beneficial effects of visual aesthetics on performance. Ergonomics, 52(10), 1311-1320.

Porat, T., \& Tractinsky, N. (2012). It's a pleasure buying here: The effects of web-store design on consumers' emotions and attitudes. Human-Computer Interaction, 27(3), 235-276.

Raila, H., Scholl, B. J., \& Gruber, J. (2015). Seeing the world through rose-colored glasses: People who are happy and satisfied with life preferentially attend to positive stimuli. Emotion, 15(4), 449.

Reber, R., Schwarz, N., \& Winkielman, P. (2004). Processing fluency and aesthetic pleasure: Is beauty in the perceiver's processing experience? Personality \& Social Psychology Review, 8(4), 364-382.

Reppa, I., \& McDougall, S. (2015). When the going gets tough the beautiful get going: aesthetic appeal facilitates task performance. Psychonomic Bulletin \& Review, 22(5), 1243-1254.

Robinson, O., Grillon, C., \& Sahakian, B. (2012). The Mood Induction Task: A standardized, computerized laboratory procedure for altering mood state in humans. Protocol Exchange, 10.

Rothermund, K., Voss, A., \& Wentura, D. (2008). Counter-regulation in affective 
attentional biases: a basic mechanism that warrants flexibility in emotion and motivation. Emotion, 8(1), 34.

Rothermund, K., Wentura, D., \& Bak, P. (2001). Automatic attention to stimuli signalling chances and dangers: Moderating effects of positive and negative goal and action contexts. Cognition \& Emotion, 15, 231-248.

Rowe, G., Hirsh, J. B., \& Anderson, A. K. (2007). Positive affect increases the breadth of attentional selection. Proceedings of the National Academy of Sciences, 104(1), 383-388.

Sanchez, A., Vazquez, C., Gomez, D., \& Joormann, J. (2014). Gaze-fixation to happy faces predicts mood repair after a negative mood induction. Emotion, 14(1), 85.

Sauer, J., \& Sonderegger, A. (2009). The influence of prototype fidelity and aesthetics of design in usability tests: effects on user behaviour, subjective evaluation and emotion. Applied Ergonomics, 40(4), 670-677.

Schindler, I., Hosoya, G., Menninghaus, W., Beermann, U., Wagner, V., Eid, M., \& Scherer, K. R. (2017). Measuring aesthetic emotions: A review of the literature and a new assessment tool. PLoS One, 12(6), e0178899.

Schmitz, T. W., De Rosa, E., \& Anderson, A. K. (2009). Opposing influences of affective state valence on visual cortical encoding. Journal of Neuroscience, 29(22), 71997207. 
Schwager, S., \& Rothermund, K. (2013). Motivation and affective processing biases in risky decision making: A counter-regulation account. Journal of Economic Psychology, 38, 111-126.

Schwager, S., \& Rothermund, K. (2014). On the dynamics of implicit emotion regulation: Counter-regulation after remembering events of high but not of low emotional intensity. Cognition \& Emotion, 28(6), 971-992.

Schwarz, N., \& Clore, G. L. (1983). Mood, misattribution, and judgments of well-being: informative and directive functions of affective states. Journal of Personality \& Social Psychology, 45(3), 513.

Sonderegger, A., \& Sauer, J. (2010). The influence of design aesthetics in usability testing: Effects on user performance and perceived usability. Applied Ergonomics, 41(3), 403-410.

Sonderegger, A., Zbinden, G., Uebelbacher, A., \& Sauer, J. (2012). The influence of product aesthetics and usability over the course of time: a longitudinal field experiment. Ergonomics, 55(7), 713-730.

Tamir, M., \& Robinson, M. D. (2007). The happy spotlight: Positive mood and selective attention to rewarding information. Personality \& Social Psychology Bulletin, 33(8), 1124-1136.

Thüring, M., \& Mahlke, S. (2007). Usability, aesthetics and emotions in humantechnology interaction. International Journal of Psychology, 42(4), 253-264. 
Tractinsky, N., Katz, A., \& Ikar, D. (2000). What is beautiful is usable. Interacting with Computers, 13(2), 127-145.

Wadlinger, H. A., \& Isaacowitz, D. M. (2006). Positive mood broadens visual attention to positive stimuli. Motivation \& Emotion, 30(1), 87-99.

Westermann, R., Spies, K., Stahl, G., \& Hesse, F. W. (1996). Relative effectiveness and validity of mood induction procedures: A meta-analysis. European Journal of Social Psychology, 26(4), 557-580. 
Table 1: Two possible mechanisms through which appeal might exert its influence on performance.

Summary of the different predicted performance outcomes with appealing versus unappealing icons for each induced mood group.

The predictions are motivated by the outcomes of studies examining the regulatory effect of emotional and mood states on

attentional processing of affective stimuli, and by evidence showing the effect of reward on mood repair. Upward arrows indicate good localisation performance. Downward arrows indicate poor localisation performance. Straight lines indicate no effect.

\begin{tabular}{|c|c|c|c|c|c|}
\hline \multirow[b]{2}{*}{$\begin{array}{l}\text { Mechanism by which } \\
\text { appeal may influence } \\
\text { performance }\end{array}$} & \multicolumn{2}{|c|}{ Positive Mood } & \multicolumn{2}{|c|}{ Negative Mood } & \multirow[b]{2}{*}{ Summary of Predictions } \\
\hline & $\begin{array}{l}\text { Appealing } \\
\text { Stimuli }\end{array}$ & $\begin{array}{l}\text { Unappealing } \\
\text { Stimuli }\end{array}$ & $\begin{array}{l}\text { Appealing } \\
\text { Stimuli }\end{array}$ & $\begin{array}{l}\text { Unappealing } \\
\text { Stimuli }\end{array}$ & \\
\hline \multirow{2}{*}{ Affect } & $\uparrow$ & $\downarrow$ & $\downarrow$ & $\uparrow$ & $\begin{array}{l}\text { Mood congruency: Better performance } \\
\text { with appealing stimuli for participants in } \\
\text { positive mood. Better performance with } \\
\text { unappealing stimuli for participants in } \\
\text { negative mood. }\end{array}$ \\
\hline & $\downarrow$ & $\uparrow$ & $\uparrow$ & $\downarrow$ & $\begin{array}{l}\text { Mood incongruency: Better performance } \\
\text { with appealing stimuli for participants in } \\
\text { negative mood. Better performance with } \\
\text { unappealing stimuli for participants in } \\
\text { positive mood. }\end{array}$ \\
\hline Reward & $\uparrow$ & - & $\uparrow$ & - & $\begin{array}{l}\text { If appealing stimuli are rewarding, they will } \\
\text { be processed preferentially by participants } \\
\text { in a positive and in a negative mood. }\end{array}$ \\
\hline
\end{tabular}


Figure captions

Figure 1: (A) Example icons used in the target localisation task. Two out of 40 icons used are shown here. (B) Example screens of all experimental trials (see Procedure for details). The placeholders were visible throughout the trial. To start each trial participants clicked the computer mouse on the OK button. This was followed by the appearance of a single target icon on the top left placeholder for 2 seconds, letting participants know which icon the target for that trial is. The target disappeared and was followed by a 9-icon array which always contained the target. The task in each trial was to click on the target icon.

Figure 2: Graph illustrating the mood ratings obtained from participants in the positive and the negative mood groups, before the induction, immediately after the induction, and at the end of the target localisation task. Error bars represent standard error of the mean.

Figure 3: Graphs illustrating the Appeal by Block interaction for each the positive $(A)$ and the negative (B) mood group. The average mood rating at block 1 is the rating obtained immediately after the mood induction, and the mood rating at block 3 is the rating obtained at the end of the experiment. Each block contained 120 trials. Error bars represent standard error of the mean. 
Mood and Aesthetic Appeal 
Mood and Aesthetic Appeal

Figure 1

(A)
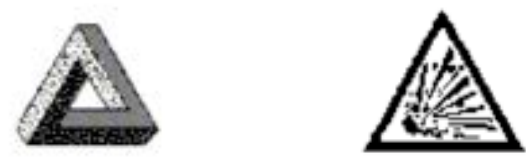

Appealing

Unappealing

(B)

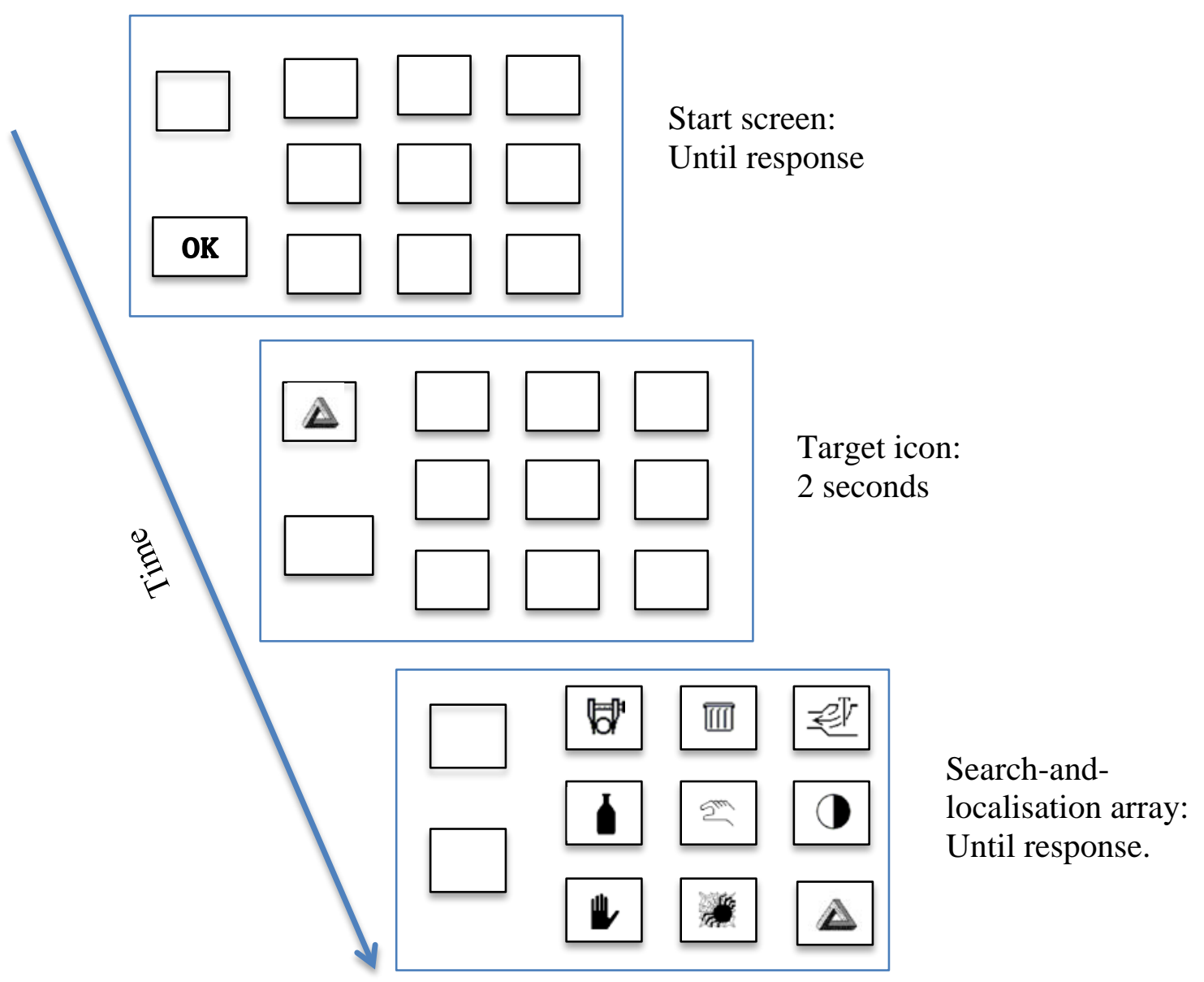


Figure 2

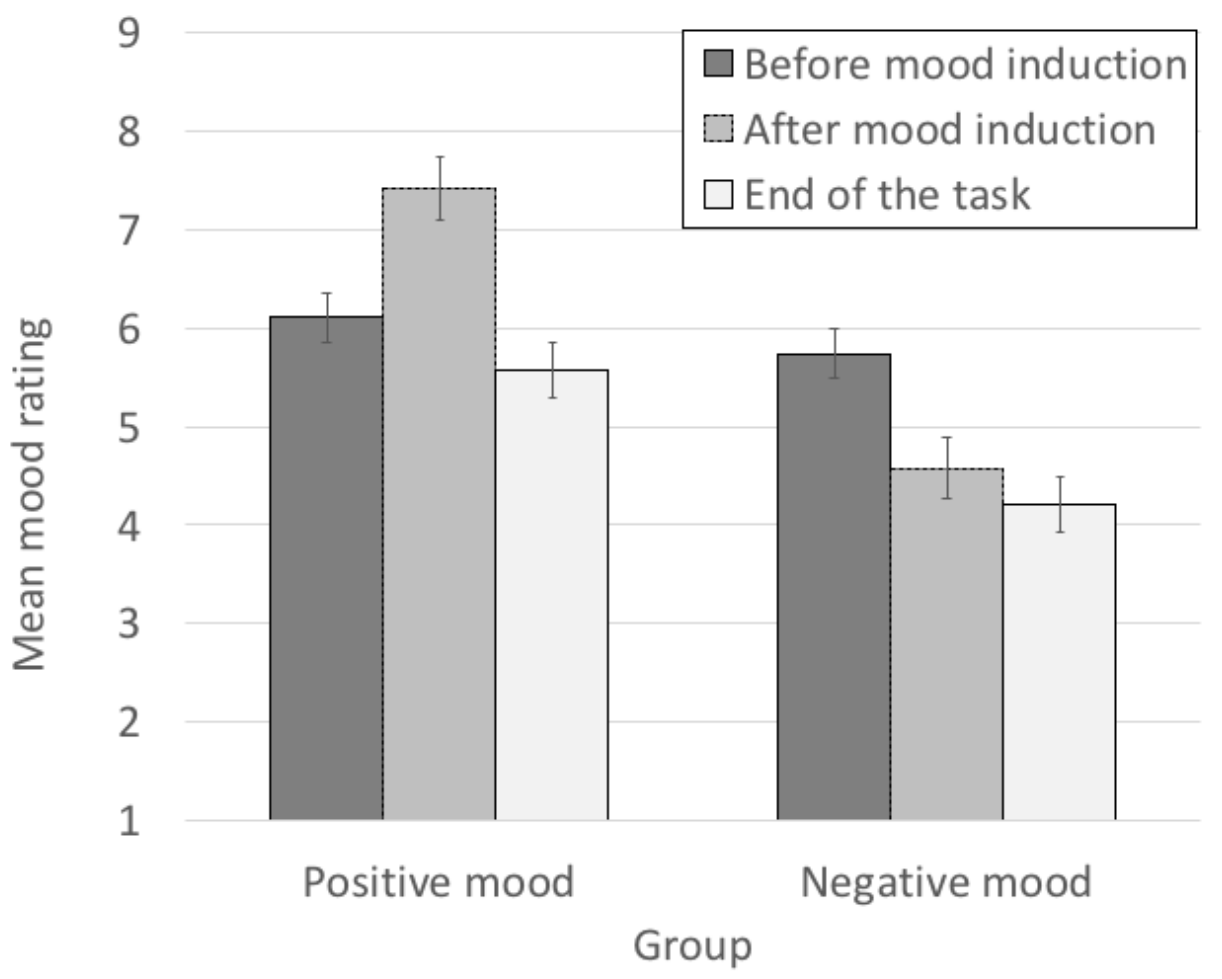


Figure 3

(A) Positive Mood

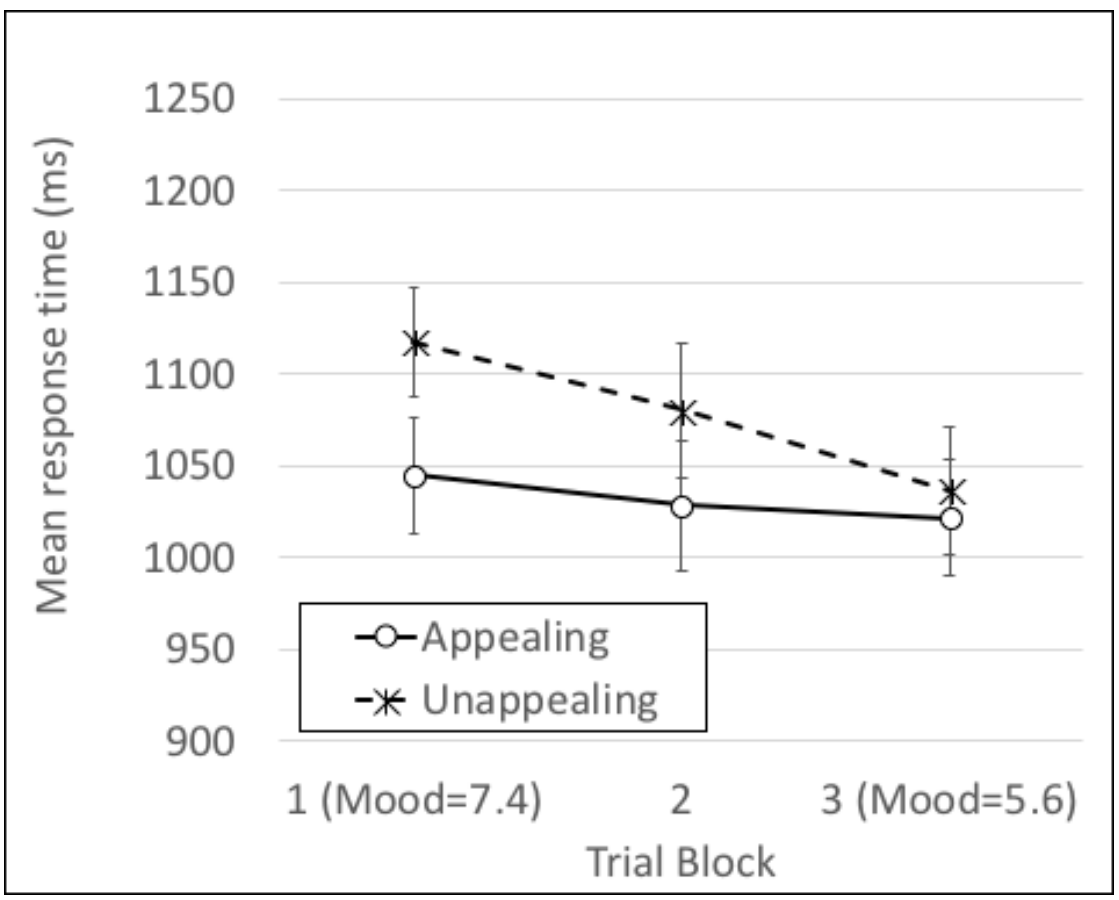

(B) Negative Mood

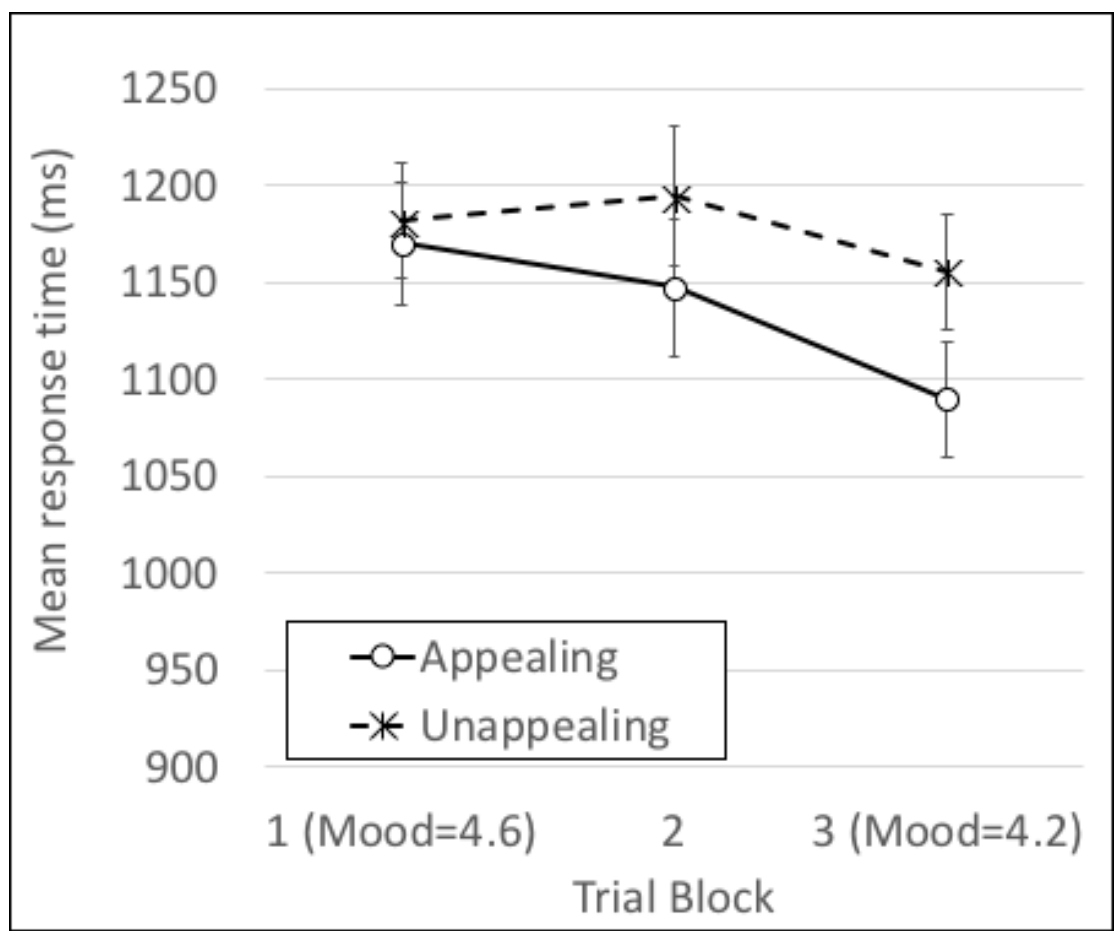


Figure 4

(A) Valence ratings

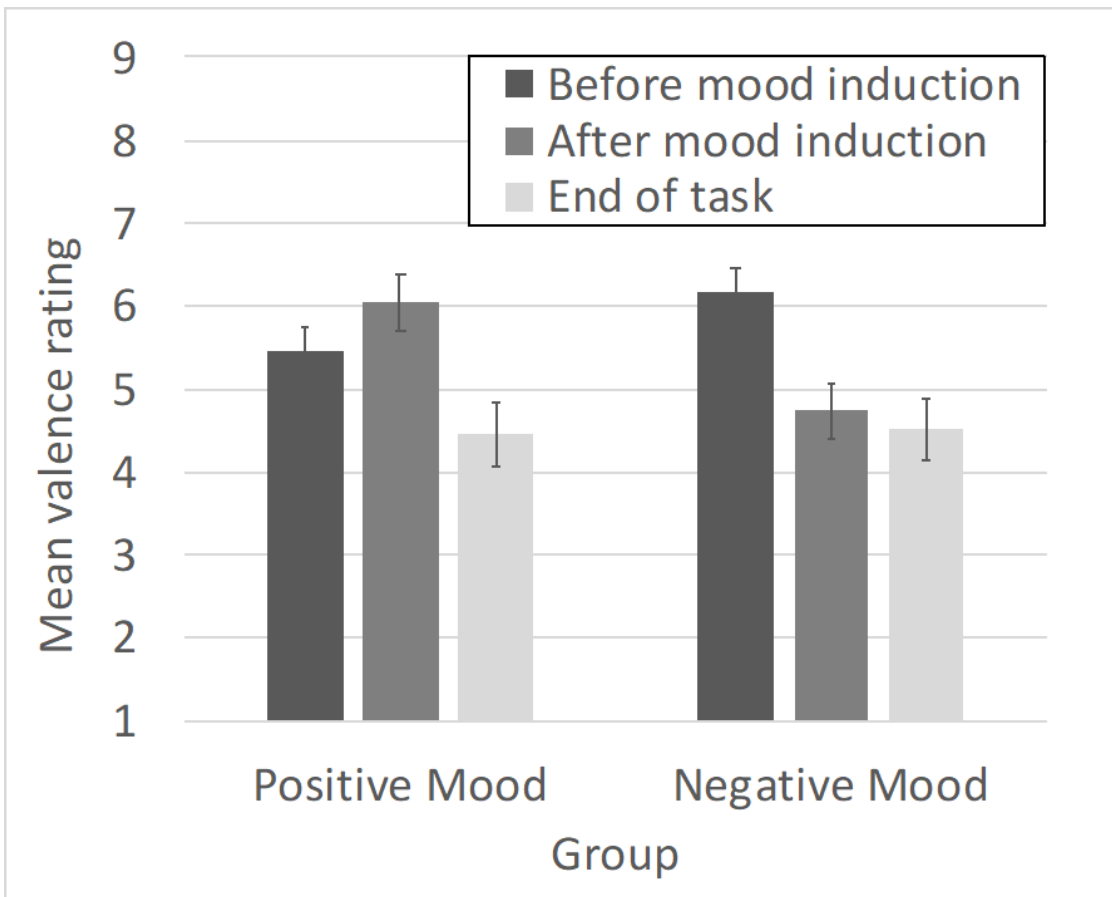


(B) Arousal ratings

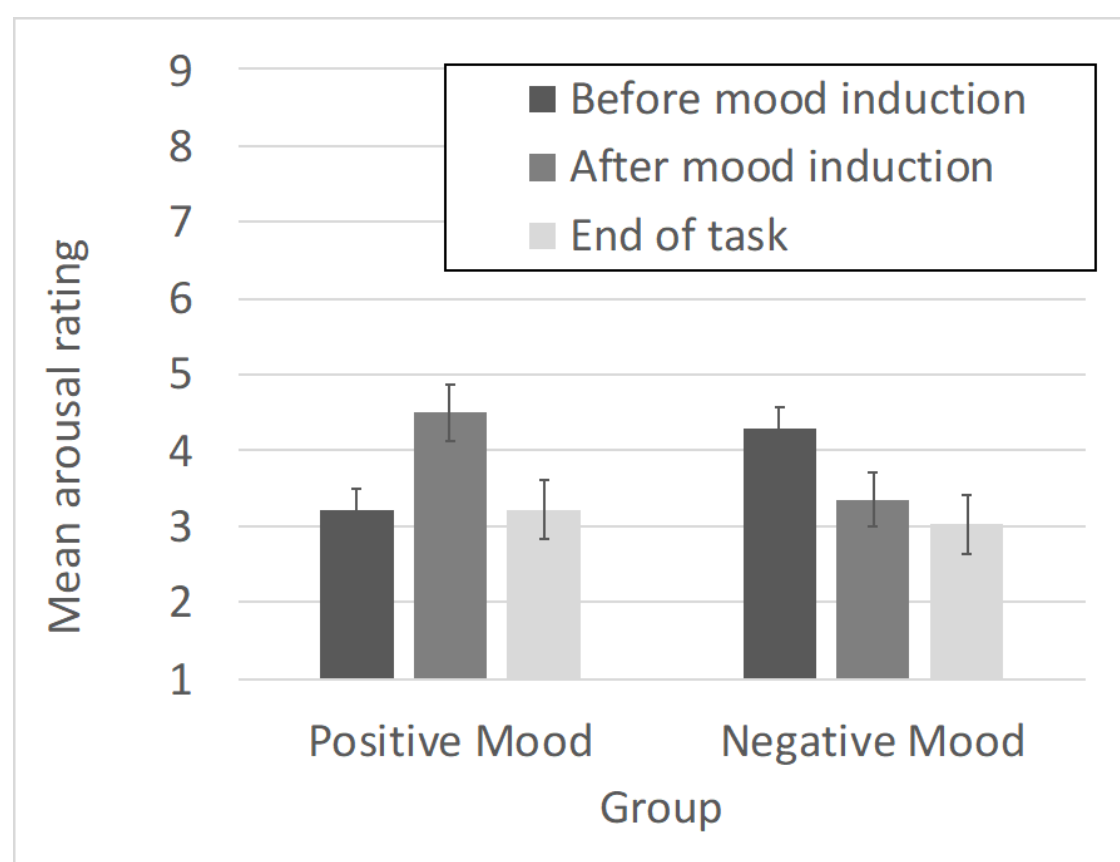

Figure 5

(A) Positive Mood

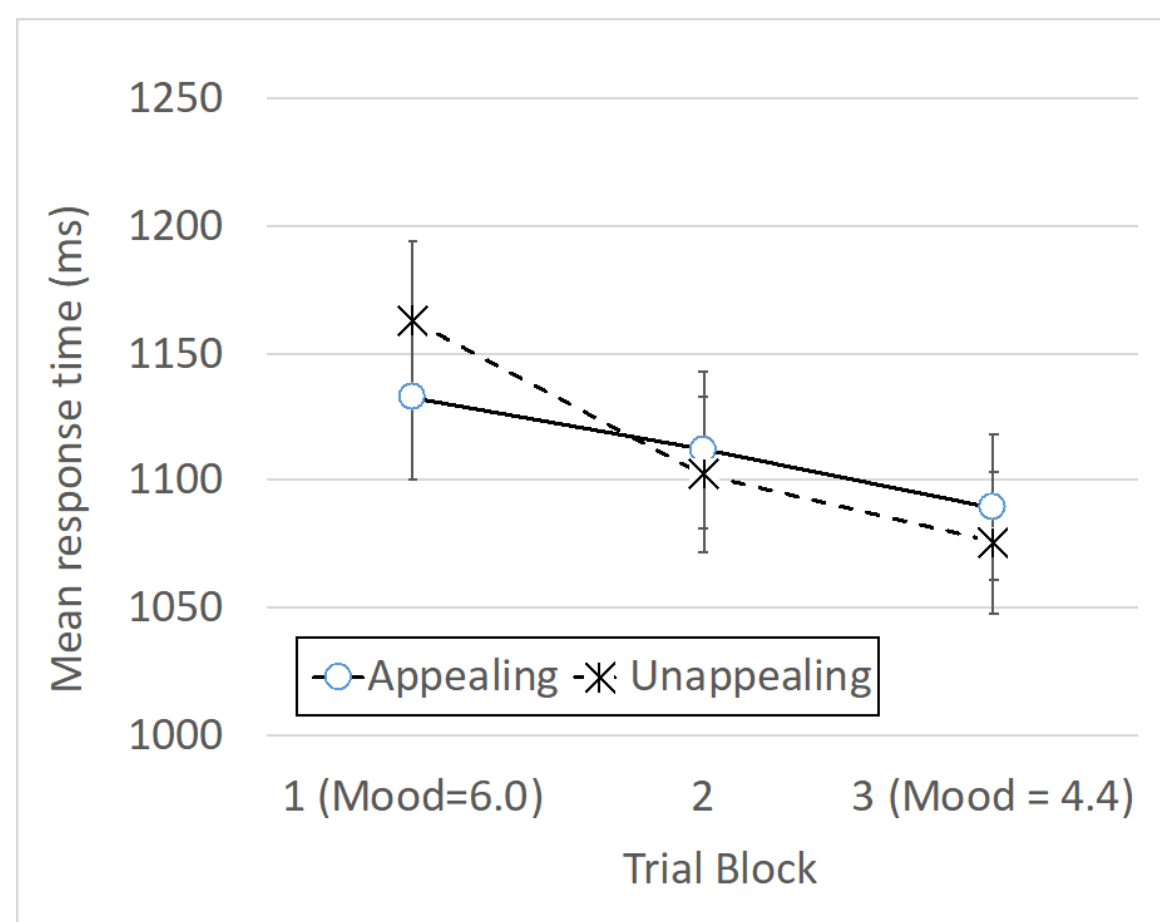

(B) Negative Mood 


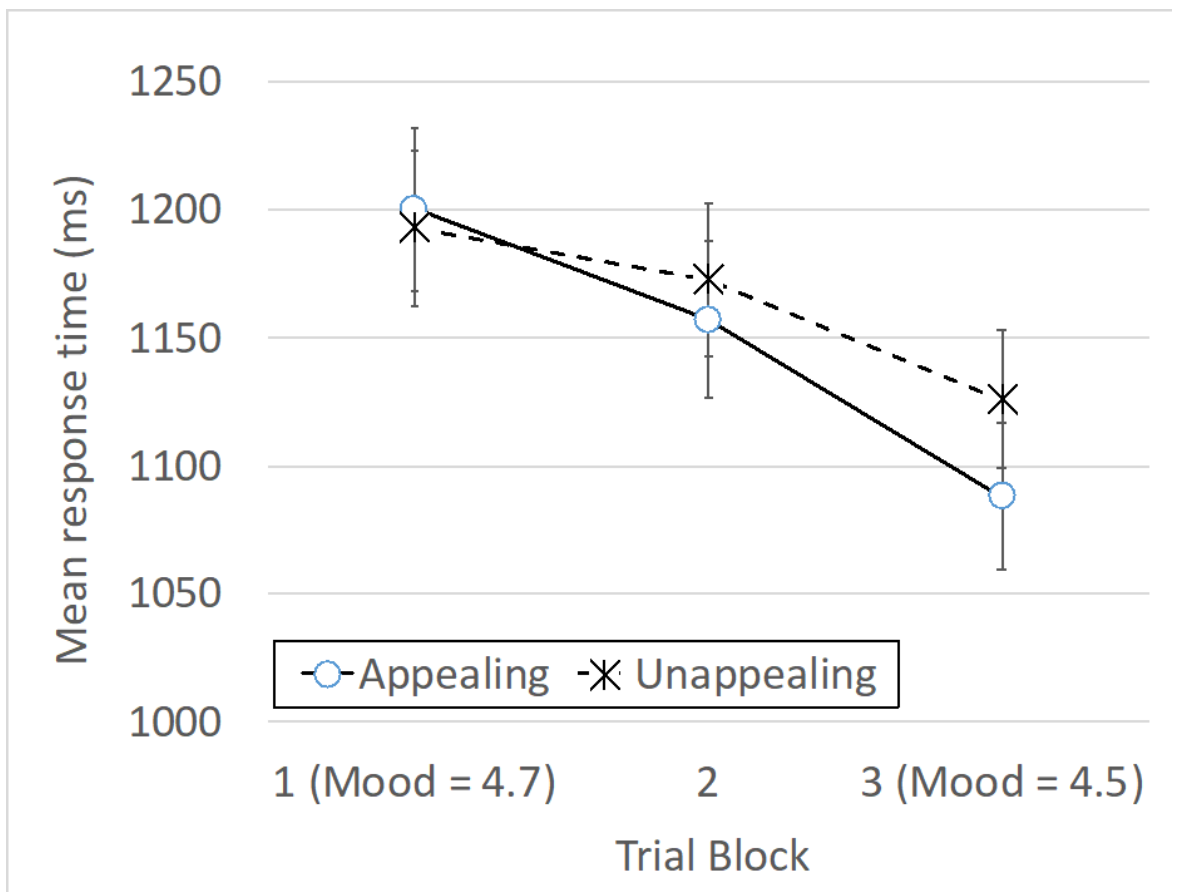


Mood and Aesthetic Appeal 